\section{La trypanosomose cameline à Trypanosoma evansi (Steel 1885), Balbiani 1888, en République islamique de Mauritanie : Résultats d'enquêtes dans le Trarza}

\author{
P. Jacquiet ${ }^{1}$ \\ M.L. Dia ${ }^{1}$ \\ D. Cheikh ${ }^{1}$
}

\section{A. 'Thiam ${ }^{1}$}

JACQUIET (P.), DIA (M.L.), CHEIKH (D.), THIAM (A.). La trypanosomose cameline à Trypanosoma evansi (Steel 1885), Balbiani 1888, en République islamique de Mauritanie : Résultats d'enquêtes dans le Trarza. Revue Élev. Méd. vét. Pays trop., 1994, 47 (1) : 59-62

Une première enquête sur la trypanosomose cameline à Trypanosoma evansi a été réalisée dans la région du Trarza (sud-ouest mauritanien). Deux cent dix-huit frottis sanguins ont été réalisés ainsi que 160 analyses sérologiques selon un test d'immunofluorescence indirecte. Les jeunes chamclons de moins d'un an scmblent épargnés par l'infection à Trypanosoma evansi, alors que chez les chamelles laitières, des taux d'infection globaux de 7,3 p. 100 (frottis sanguins) et 24,5 p. 100 (sérologie) ont été trouvés. Le mode de conduite du troupeau semble être un facteur de risque important : ce sont les troupeaux qui fréquentent, même temporairement, les zones boisées de la vallée du fleuve Sénégal ou des collections d'eau permanentes (lac de R'kiz) qui présentent les plus forts taux d'infection. Des recommandations aux éleveurs sont proposées, notamment pour les troupeaux de chamelles laitières du sud du pays.

Mots clés : Dromadaire - Camelus dromedarius - Trypanosomose - Trypanosoma evansi - Enquête pathologique - Sérologie - Immunofluorescence indirecte - Facteur de risque - Conduite du troupeau - Mauritanie.

\section{Introduction}

L'élevage du dromadaire est pratiqué dans la quasi-totalité des zones habitées de la République islamique de Mauritanie. On estime, en 1993, le cheptel mauritanien à 1000000 de têtes (en 1992, l'Annuaire FAO de la Production cite le chiffre de 990 000) ce qui, numériquement, le place largement au premier rang des cheptels camelins d'Afrique de l'Ouest. Dans de nombreux pays, la trypanosomose à Trypanosoma evansi a été identifiée comme pathologie dominante dans l'élevage camelin $(7,8,9,10$, $11,13,15,16,22$ ). Paradoxalement, elle a fait l'objet de peu d'études en Mauritanie (4). Cette note présente les premiers résultats d'enquêtes effectuées dans la région du Trarza (sud-ouest mauritanien) entre 1990 et 1992.

\section{Matériels et méthodes}

\section{Le milieu}

L'étude s'est déroulée dans le Trarza, région du SudOuest, que l'on peut diviser en deux grandes zones:

1. Centre national d'élevage et de recherches vétérinaires, Service de parasitologie, BP 167, Nouakchott, Mauritanie.

Reçu le 27.4.1993, accepté le 27.01.1994.
- la vallée du fleuve Sénégal et les collections d'eau permanentes du Sud (lac de R'kiz) ;

- les systèmes dunaires, constitués de massifs dunaires en remobilisation éolienne permanente et de dépressions interdunaires. Les systèmes dunaires couvrent plus de 95 p. 100 de la région.

La principale caractéristique du climat est l'existence d'une longue saison sèche, d'octobre à juillet, et d'une très courte saison des pluies centrée sur le mois d'août. La vallée du fleuve Sénégal appartient au domaine sahélien entre les isohyètes 150 et $250 \mathrm{~mm}$. Consacrée en grande partie à l'agriculture (mise en valeur de la vallée du fleuve), elle constitue néanmoins une zone refuge pour le bétail, notamment en période de soudure d'avril à juillet. Le reste de la zone d'étude $(50$ à $150 \mathrm{~mm}$ de pluies annuelles) présente un couvert ligneux clairsemé : Balanites aegyptiaca et Acacia senegal, exploité par les camelins. Le couvert herbacé a vu la régression des graminées sahéliennes de bonne valeur fourragère, au profit d'espèces peu appétées.

L'élevage camelin dans le Trarza est de type traditionnel, extensif. Depuis quelques années, on assiste à une concentration de chamelles laitières autour des centres urbains (Nouakchott, Rosso). On peut distinguer trois types de conduite de troupeaux dans la zone:

- des troupeaux qui restent confinés toute l'année dans la vallée du fleuve Sénégal (groupe 1);

- ceux qui ne descendent sur le fleuve qu'en période de soudure (groupe 2) ;

- ceux qui ne descendent jamais dans la vallée du fleuve Sénégal pour fuir les zones à "mouches" (groupe 3).

L'enquête a porté sur trois à quatre troupeaux de chacun de ces groupes. Une vingtaine d'animaux ont été examinés et des prélèvements de sang ont été effectués dans chaque troupeau.

\section{Mise en évidence des parasites sur frottis sanguins}

Le sang prélevé sur anticoagulant (EDTA) sert pour la réalisation de frottis sanguins colorés au Giemsa, ainsi que pour la mesure de l'hématocrite (microcentrifugeuse de terrain Compur 1101). Les lames sont ensuite observées au grossissement 400 .

\section{Sérologie}

Le sang est prélevé sur un tube siliconé pour la collecte de sérum. L'antigène $T$. evansi provient d'une souche isolée sur une chamelle laitière de la vallée du fleuve Sénégal en juillet 1990, et entretenue depuis sur lapin. La réalisation des lames d'antigène nécessitant une parasitémie élevée, l'inoculation préalable à la souris a 
été pratiquée. La méthode sérologique employée est un test d'immunofluorescence utilisant la fixation du complément humain ICFT (24).

\section{Résultats}

\section{Lecture des frottis sanguins (tableau I)}

Sur 218 frottis réalisés, 16 sont positifs. Trypanosoma evansi est présent dans tous les cas positifs. Un seul frottis fait apparaître une infection mixte $T$. evansi et $T$. vivax. Les parasitémies sont très variables : de quelques trypanosomes par lame à 15 par champ microscopique. C'est dans le groupe 1 que l'on rencontre le plus fort taux de frottis positifs : 15 p. 100 contre 5 p. 100 environ pour les groupes 2 et 3 . On constate que $T$. evansi n'a été décelé chez aucun chamelon.

TABLEAUI Lecture des frottis sanguins.

\begin{tabular}{|l|l|c|c|}
\hline Groupe & Age & $\begin{array}{c}\text { Nombre d'animaux } \\
\text { examinés }\end{array}$ & $\begin{array}{c}\text { Pourcentage de } \\
\text { frottis positifs }\end{array}$ \\
\hline Groupe 1 & $\begin{array}{l}\text { Adultes } \\
\text { Chamelons }\end{array}$ & $\begin{array}{r}67 \\
8\end{array}$ & $\begin{array}{c}14,9 \\
0\end{array}$ \\
\hline Groupe 2 & Adultes & 78 & 5,1 \\
& Chamelons & 11 & 0 \\
\hline Groupe 3 & Adultes & 45 & 4,4 \\
& Chamelons & 9 & 0 \\
\hline Total & & 218 & 7,34 \\
\hline
\end{tabular}

\section{Sérologies en ICFT (tableau II)}

Cent soixante sérums ont été testés. La séro-prévalence est forte dans les groupes 1 et 2 (respectivement 38 et 30 p. 100); en revanche, elle est plus faible dans le groupe $3: 11$ p. 100. Si l'on compare les résultats des groupes 1 et 2, la différence n'est pas significative; en revanche, elle l'est pour les groupes 1 et 3 ou 2 et 3 : $P<0,01$ et $P<0,05$, respectivement. On note que sur 20 chamelons testés, un seul est positif (groupe 2) et un seul est douteux (groupe 1) pour 18 négatifs.

\section{Incidence clinique}

Au cours de l'enquête, une seule chamelle a présenté une affection aiguë : amaigrissement net avec effacement de la bosse, apathie (l'animal reste à l'arrière du troupeau), conjonctivite et larmoiement. La lecture des hématocrites (tabl. III) révèle souvent une anémie prononcée (hématocrite compris entre 10 et 15), notamment chez des chamelles à sérologie positive. On note également une corrélation nette entre sérologies positives et commémoratifs d'avortements répétés.
TABLEAU III Comparaison des hématocrites entre dromadaires à sérologies positive et négative.

\begin{tabular}{|l|c|c|}
\hline & $\begin{array}{c}\text { Nombre d'animaux } \\
\text { examinés }\end{array}$ & $\begin{array}{c}\text { Hématocrite } \\
\text { moyen }\end{array}$ \\
\hline $\begin{array}{l}\text { Dromadaires à sérologie } \\
\text { positive }\end{array}$ & 18 & 21 \\
\hline $\begin{array}{l}\text { Dromadaire à sérologie } \\
\text { négative }\end{array}$ & 48 & 26 \\
\hline
\end{tabular}

\section{Discussion}

Ces résultats, limités à la seule région du Trarza, sont comparables à ceux obtenus par CHRISTY (4) en 19871988 à l'abattoir de Nouakchott : 5,8 p. 100 de frottis positifs sur 172 réalisés. De même, DIALL (7), dans une enquête dans le nord-ouest malien sur des troupeaux transhumants en provenance de Mauritanie, a mis en évidence un taux d'infection global de 10 p. 100 sur 767 frottis réalisés (7). La trypanosomose cameline à $T$. evansi est donc bien répandue en République islamique de Mauritanie.

La sérologie ICFT fait apparaître un plus fort taux d'infection que la lecture des frottis sanguins dont le manque de sensibilité a été signalé par de très nombreux auteurs (14, $17,18,19,20,21,23,26,27)$. L'étude des premiers résultats indique qu'il existerait une corrélation entre la prévalence de l'infection à $T$. evansi et la fréquentation des zones boisées proches de cours d'eau ou de collections d'eau permanente (vallée du fleuve Sénégal et lac de $\left.R^{\prime} k i z\right)$. Ceci peut s'expliquer par une plus grande abondance de Tabanidés et/ou d'Hippoboscidés, vecteurs qui trouvent dans ces régions à la fois des gîtes de reproduction, de repos et du bétail pour se nourrir. Il faut noter cependant que dans le groupe 3 la séro-prévalence n'est pas nulle (11 p. 100). L'existence d'une transmission, même faible, dans les oasis du nord mauritanien ne peut être totalement exclue mais il est vraisemblable que les mouvements commerciaux entre les groupes sont en partie responsables de ce taux de séro-prévalence non négligeable.

Les résultats trouvés ici, ainsi que ceux de CHRISTY (4) et DIALL (7), ne font pas apparaître de frottis sanguins positifs chez les chamelons de moins d'un an, même dans les régions de forte endémicité. En ICFT, on retrouve une très faible prévalence de $T$. evansi : 5 p. 100. S'agit-il d'une résistance naturelle des chamelons, d'une moindre attractivité pour les insectes vecteurs ou, plus simplement, du résultat d'une incidence annuelle faible, les chamelons n'ayant pas encore eu le temps d'être infectés ? Cette question mérite de plus amples investigations. Quant à l'identification d'une infection naturelle par $T$. vivax, rarement rapportée dans la bibliographie, elle a été réalisée uniquement sur une base morphologique et mérite aussi une confirmation ultérieure. 
TABLEAU II Résultats des sérologies ICFT.

\begin{tabular}{|c|c|c|c|c|c|}
\hline \multirow{2}{*}{ Groupe } & \multirow{2}{*}{ Age } & \multirow{2}{*}{$\begin{array}{l}\text { Nombre d'animaux } \\
\text { examinés }\end{array}$} & \multicolumn{3}{|c|}{ Sérologie } \\
\hline & & & positive & douteuse & négative \\
\hline Groupe 1 & $\begin{array}{l}\text { Adultes } \\
\text { Chamelons }<1 \text { an }\end{array}$ & $\begin{array}{l}58 \\
8\end{array}$ & $\begin{array}{c}22 \\
(38 \%) \\
0\end{array}$ & $\begin{array}{c}11 \\
(19 \%) \\
1\end{array}$ & $\begin{array}{c}25 \\
(43 \%) \\
7\end{array}$ \\
\hline Groupe 2 & $\begin{array}{l}\text { Adultes } \\
\text { Chamelons }<1 \text { an }\end{array}$ & $\begin{array}{l}37 \\
11\end{array}$ & $\begin{array}{c}11 \\
(29,7 \%) \\
1\end{array}$ & $\begin{array}{c}5 \\
(13,5 \%) \\
0\end{array}$ & $\begin{array}{c}21 \\
(56 \%) \\
10\end{array}$ \\
\hline Groupe 3 & $\begin{array}{l}\text { Adultes } \\
\text { Chamelons }<1 \text { an }\end{array}$ & $\begin{array}{c}45 \\
1\end{array}$ & $\begin{array}{c}5 \\
(11 \%) \\
0\end{array}$ & $\begin{array}{c}10 \\
(22 \%) \\
0\end{array}$ & $\begin{array}{c}30 \\
(67 \%) \\
1\end{array}$ \\
\hline Total & Tous âges & 160 & $\begin{array}{c}39 \\
(24,4 \%)\end{array}$ & $\begin{array}{c}27 \\
(16,9 \%)\end{array}$ & $\begin{array}{c}94 \\
(58,7 \%)\end{array}$ \\
\hline
\end{tabular}

\section{Conclusion}

La trypanosomose à $T$. evansi constitue une contrainte majeure de l'élevage camelin dans le Sud-Trarza. Responsable d'une diminution des productions de viande et de lait, elle semble également liée aux avortements répétés constatés chez de nombreuses chamelles de la zone. Un contrôle strict de cette trypanosomose utilisant des trypanocides à activité chimioprophylactique (chlorure et sulfate de quinapyramine par exemple) semble nécessaire pour les troupeaux de chamelles laitières sédentaires, autour des centres urbains du sud du pays: Rosso, R'kiz... Ces trypanocides chimioprophylactiques pourraient également être administrés aux troupeaux qui transhument vers le sud deux ou trois mois par an et ce, avant leur arrivée dans les zones susceptibles d'abriter des populations importantes d'insectes hématophages.

\section{Remerciements}

Ce travail a reçu l'aide financière de la Mission de Coopération française en Mauritanie. Les auteurs tiennent également à remercier $\mathrm{M}$. EI MABROUK $\mathrm{O}$. EI M'REIZIG pour son aide technique ainsi que les éleveurs de dromadaires mauritaniens qui ont bien voulu mettre leurs animaux à leur disposition.

\section{Bibliographie}

1. ALEMU (T.). Camel trypanosomiasis in Ethiopia. $n$ : Premier séminaire international sur les trypanosomoses animales non transmises par les glossines, Annecy, France, octobre 1992.

2. BAJYANA SONGA (E.), KAGERUKA (P.). HAMERS (R.). The use of the card agglutination test (Testryp ${ }^{\mathrm{ND}}$ CATT) for the serodiagnosis of $T$. evansi infections. Annls Soc. belge Med. trop., 1987, 67: 51-57.
3. RAJYANA SONGA (E.), HAMERS (R.). A card agglutination test (CATT) for veterinary use based on an early Vat Rotat $1 / 2$ of Trypanosoma evansi. Annls Soc. belge Med. trop., 1988, 68: 233-240.

4. CHRISTY (P.). Convention IEMVT/CNERV. Rapport d'activités 1987. Nouakchott, CNERV.

5. CURASSON (G.). Traité de protozoologie vétérinaire et comparée. Tome 1. Trypanosomes. Paris, Vigot frères éd., 1943. 445 p.

6. DIA (M.L.). Comparaison du pouvoir pathogène de la souche Trypanosoma evansi de Mauritanie avec celles du Kenya, du Niger, du Tchad et de la Chine. In : Premier séminaire international sur les trypanosomoses animales non transmises par les glossines, Annecy, France, octobre 1992

7. DIALL (O.). Enquête sur la trypanosomose cameline dans les États du CILSS. Rapport préparé pour le Bureau régional de la FAO pour l'Afrique. Rome, Accra, Ghana, FAO, juillet 1991. 35 p.

8. DIRIE (M.F.). WALLBANKS (K.A.), ADEN (A.A.), BORNSTEIN (S.). IBRAHIM (M.O.). Camel trypanosomiasis and its vectors in Somalia. Vet. Parasitol.., 1989. 32: 285-291.

9. ELDISOUGI (M.). A note on diseases of camels in Saudi Arabia. In : COCKRILL (W.R.) éd. The camelid: an all purpose animal. Proceedings of the Khartoum Workshop on camels. December 1979. Uppsala, Scandinavian Institute of African Studies. 1984.

10. GODFREY (D.G.). KILLICK-KENDRICK (R.). Trypanosoma evansi of camels in Nigeria: a high incidence demonstrated by the inoculation of blood into rats. Annls trop. Med. Parasit. 1961, 56: 14-19.

11. GRUVEL (J.), BALIS (J.). La trypanosomiase à Trypanosoma evansi chez le dromadaire au Tchad et ses principaux vecteurs. Revue Élev. Méd. vét. Pays trop., 1965, 18 (4) : 435-439.

12. HOARE (C.A.). The trypanosomes of mammals: a zoological monograph. London, Blackwell Scientific Publications, 1972.749 p.

13. LUSOS (G.J.). Diseases caused by Trypanosoma evansi: a review. Vet. Res. Commun. 1980, 4: 165-181.

14. LUCKINS (A.G.), BUID (R.), RAE (P.), MAHMOUD (M.M.), EL MALIK (K.H.). GRAY (A.R.). Serodiagnosis of infection with Trypanosoma evansi in camels in the Sudan. Trop. Anim. Hlth Prod.. 1979, 11: 1-12.

15. MAHMOUD (M.M.). OSMAN (M.O.). A note on trypanosomiasis in Sudan camels. $n$ : COCKRILL (W.R.) éd. The camelid: an all purpose 


\section{Communication}

animal. Proceedings of the Khartoum Workshop on camels, December 1979. Uppsala, Scandinavian Institute of African Studies, 1984

16. MAHMOUD (M.M.), GRAY (A.R.). Trypanosomiasis due to Trypanosoma evansi (Steel 1885), Balbiani 1888. A review of recent research. Trop. Anim. Hlth Prod., 1980, 12: 35-47.

17. MORGAN (E.S.), BENETT (S.C.J.). The mercuric chloride test for trypanosomiasis in camels: mechanism of the reaction. J. comp. Path. Ther. $1929,42(3)$ : 188-196.

18. NANTULYA (V.M.). Trypanosomiasis in domestic animals: the problem of diagnosis. Revue sci, tech. Off. int. Epizoot., 1990, 9 (2) : 357-367.

19. NANTULYA (V.M.), BAJYANA SONGA (E.), HAMERS (R.) Detection of circulating trypanosomal antigens in Trypanosoma evansiinfected animals using a $T$. brucei group-specific monoclonal antibody. Trop. Med. Parasit., 1989, 40: 263-266.

20. NANTULYA (V.M.), LINDQUIST (K.J.). Antigen detection enzyme immuno-assays for the diagnosis of Trypanosoma vivax, T. congolense and T. bracei infections in callle. Trop. Med. Parasil., 1989, 40: 267-272.

21. NANTULYA (V.M.), LINDQUIST (K.J.), DIALL (O.), OLAHO MUKANI (W.). Two simple antigen-detection enzyme inmuno-assays for the diagnosis of Trypanosoma evansi infections in the dromedary camel (Camelus dromedarius) Trop. Med. Parasit., 1989, 40: 415-418.

22. OLAHO-MUKANI (W.), LUCKINS (A.G.), MUTUGI (M.W.), OMUSE (J.K.), SAYER (P.D.), KIMANI (J.). Recent observations on the prevalence of Trypanosona evansi infection in camels in Kenya. $I n$ : Pre mier séminaire international sur les trypanosomoses animales non transmises par les glossines, Annecy, France, octobre 1992.

23. PEGRAM (R.G.), SCOTT (J.M.). The prevalence and diagnosis of Trypanosoma evansi infection in camel in Southern Ethiopia. Trop. Anim. Hlth Prod., 1976, 8: 20-27.
24. PERIE (N.M.), TINNEMANS-ANGGAWIDJAJA (T.), ZWART (D.) A refinement of the immunofluorescent complement fixation test for Trypanosoma infections. Tropenmed. Parasit. 1975, 25: 399-404.

25. SCHWARTZ (M.J.), DIOLI (M.). The one-humped camel in eastern Africa: a pictorial guide to diseases, health care and management. Weikersheim, Verlag Josef Margraf, 1992. 282 p.

26. VERY (P.), BOCQUENTIN (R.), DUVALLET (G.). Sensibilité de la double centrifingation pour la recherche des trypanosomes. Revue Elev. Méd. vét. Pays trop., $1990, \mathbf{4 3}$ (3) : 325-329.

27. ZWEYGARTH (E.), SABWA (C.), RÖTTCHER (D.). Serodiagnosis of trypanosomiasis in dromedary camels using a card agglutination test set (Testryp ${ }^{N D}$ CATT). Annls Soc. belge Med. trop., 1984, 64: 309-313.

JACQUIET (P.), DIA (M.L.), CHEIKH (D.), THIAM (A.). Camel trypanosomosis due to Trypanosoma evansi (Steel 1885), Balbiani 1888, in Mauritania : Results of surveys in the 'Irarza region. Revue Élev. Méd. vét. Pays trop., 1994, 47 (1) : 59-62

A first survey concerning camel trypanosomosis due to Trypanosoma evansi was carried out in the Trarza region (south-western Mauritania). Blood smears were made from 218 animals and 160 were submitted to an indirect immunofluorescence test. Young calves below one year old seem to be free of $T$. evansi infection, while in dairy females, average rates of infection of $7.3 \%$ (blood smears) and $24.5 \%$ (serological results) were found. The type of herd management seems to be an important risk factor: the herds that frequent, even temporarily, the wooded areas of the Senegal river valley or permanent water collections (R'kiz lake) show the highest infection rates. Recommendations are presented to camel rearing owners, especially those with dairy camel herds in the South of the country.

Key words : Dromedary - Camelus dromedarius - Trypanosomosis - Trypanosoma evansi - Pathological survey - Serology - Indirect immunofluorescence - Risk factor - Livestock management - Mauritania. 